\title{
Experimental Study on Cartesian-Space PD Control for Hydraulic Manipulator
}

\author{
Sang-Uk Chon, Jung-San Cho, and Sang-Deok Park
}

\begin{abstract}
This paper presents Cartesian-space PD control of a hydraulic manipulator. The approach based upon "Virtual spring-damper hypothesis" is composed of virtual spring effects and virtual damper effects in task space. It has been applied to electrically driven robots. This experiment shows the comparison of Cartesian-space control with typical joint-space control in the performance of straight-line motion for the hydraulic manipulator that easily generate movements of manipulator without spending a huge amount of computational cost.
\end{abstract}

Index Terms-Cartesian-space control, hydraulic actuator, manipulator, position control.

\section{INTRODUCTION}

Robot manipulators are highly required in many kinds of industrial tasks not only electrically driven robots but also hydraulically driven robots such as spray painting, arc welding, excavator and surgery for human. Among several tasks to be automated, one may regard straight-line motion as one of the most fundamental and essential task. In order to automate straight-line motion, Cartesian-space control must be used. In other words, the end-effector of the manipulator needs to be controlled to track a linear path on the task space [1].

There are two types of robots in usual. One is electrically driven robots and the other is hydraulically driven robot. Both of them have characteristics. Model-based control algorithms have been studied in electrically driven robots to improve position and tracking performance compared to classical approaches. There are some advantages in hydraulic driven robots those are the high power-to-weight ratio, the stiffness and the short response time. In particular, the hydraulically driven manipulators, being massively coupled and complexly connected and having at the same time various nonlinear components, contain numerous forms of severe nonlinearities that are hardly observed in electrically driven robots. As a result, the end-effector control in task space of a hydraulically driven manipulator becomes significantly more difficult than

Manuscript received January 15, 2014; revised March 21, 2014. This work was supported by the Industrial Strategic technology development, 10047635, Development of Hydraulic Robot Control Technology based on Accurate and Fast Force Control for Complex Tasks funded By the Ministry of Trade, Industry \& Energy (MI, Korea)

Sang-Uk Chon is with Intelligent Robot Engineering, University of Science \& Technology, 176 Gajung-dong 217 Gajungro Yuseong-gu, Daejeon, Korea and Department of Applied Robot Technology, Korea Institute of Industrial Technology, 1271-18 Sa3-dong, Sangrok-gu, Ansan, Korea (e-mail: jsoman00@gmail.com)

Jung-San Cho and Sang-Deok Park are with the Department of Applied Robot Technology, Korea Institute of Industrial Technology, 1271-18 Sa3-dong, Sangrok-gu, Ansan, Korea (e-mail: chojs,sdpark@kitech.re.kr) the Cartesian-space control of a typical robot driven by electric motors [2].

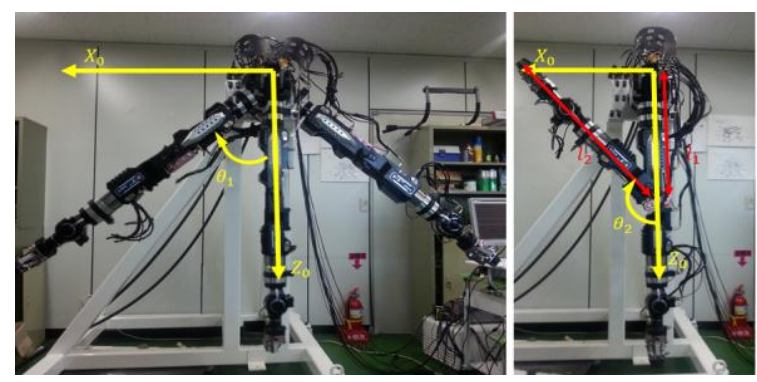

Fig. 1. Geometrical relation of 2-DOF planar manipulator.

\section{ANALYSIS OF MANIPULATOR}

\section{A. Manipulator Part}

In this paper, only two joints are used and others are fixed by position control not considered for straight-line motion. Then $Y$ axis is neglected in this experiment. The 2-DOF planar manipulator is shown in Fig. 1. Since the revolute axes are all parallel, and all links are lying in the plane $\left(X_{0}, Z_{0}\right)$.

The external hydraulic power supplies pressure to this manipulator with electrical servo valves. Each links and joints geometric parameters are shown in Table I. The joints are equipped with rotary potentiometers to measure angle of joints.

\section{B. Actuator Part}

A model for the rigid body dynamics for hydraulic actuator is as following:

$$
m \ddot{x}=p_{2} A_{2}-p_{1} A_{1}-g(y, \dot{y})
$$

where $m$ is the mass of the piston and load, $p_{1}$ and $p_{2}$ are the pressures on chamber 1 and 2 of the cylinders shown in Fig. 2. $A_{1}$ and $A_{2}$ are the areas of sides 1 and 2. In this experiment, the manipulator is equipped with double rod linear actuator and rotary actuator; then $A_{1}$ and $A_{2}$ are same and neglected. $g(y, \dot{y})$ is the force of Coulomb friction, stiction, and viscous friction.

TABLE I: GEOMETRIC PARAMETERS

\begin{tabular}{cccc}
\hline \hline Symbol & Max. (Degree) & Min. (Degree) & Length $(\mathrm{m})$ \\
\hline$\theta_{1}$ & 50 & -50 & - \\
$\theta_{2}$ & 135 & 0 & - \\
$l_{1}$ & - & - & 0.59 \\
$l_{2}$ & - & - & 0.91 \\
\hline \hline
\end{tabular}




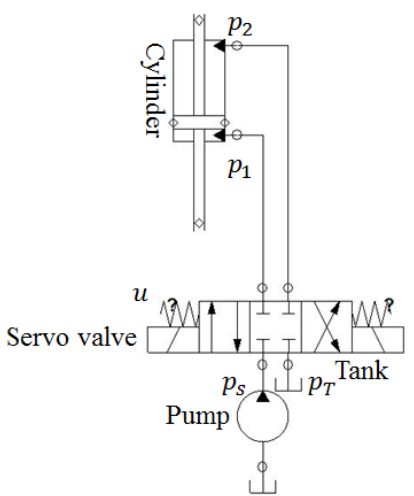

Fig. 2. Structure of the hydraulic actuator with servo valve.

We assume that the input current $u$ applied to the servo valve is directly proportional to the spool position. The dynamics of the servo valve motor and flapper are assumed to be fast enough to be neglected. The fluid flow rates $q_{1}$ and $q_{2}$ into sides 1 and 2 of the cylinder depend on the sign of the current

$$
q_{1}= \begin{cases}u c_{1} \sqrt{p_{s}-p_{1}} & \text { for } u \geq 0 \\ u c_{2} \sqrt{p_{1}-p_{T}} & \text { for } u<0\end{cases}
$$

and

$$
q_{2}= \begin{cases}-u c_{2} \sqrt{p_{2}-p_{T}} & \text { for } u \geq 0 \\ -u c_{1} \sqrt{p_{s}-p_{1}} & \text { for } u<0\end{cases}
$$

where $p_{s}$ is the supply pressure, $p_{T}$ is the tank pressure, $c_{1}$ is the valve charge coefficient and $c_{2}$ is the valve discharge coefficient [3]. The input current of hydraulic actuator is related to the output of fluid flow rates $q$ not a torque.

\section{CONTROLLER DESIGN}

\section{A. Joint-Space Position Controller}

A manipulator motion is typically assigned in the operational space in terms of trajectory parameters such as the initial and final end-effector location, possible intermediate locations, and traveling time along particular geometric paths. If it is desired to plan a trajectory in the Joint-Space, the values of the joint variables have to be determined first from the end-effector position and orientation specified by the user. It is then an inverse kinematics algorithm needed to generates functions of time sequence of values for the joint variables $q(t)$ for each joint [4]. In this paper, we can get the inverse kinematics of 2DOF planar manipulator easily by geometric method. The resulting end-effector motion is not easily predictable, in view of the nonlinear effects introduced by direct kinematics. Then real joint variables from sensors and desired joint variables from the inverse kinematics would be used for simple proportional-derivative controllers for each joint.

\section{B. Cartesian-Space PD Controller}

Arimoto et al. have proposed a simple control scheme of the Task-Space feedback based on Virtual Spring-Damper Hypothesis for electrically driven robot arm as seen in Fig. 3.

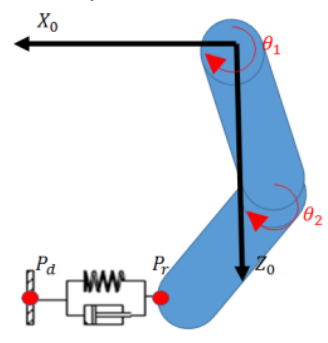

Fig. 3. Virtual spring-damper hypothesis.

The virtual work $(W)$ generated by force $(f)$ in Cartesian-space and torque $(\tau)$ in joint-space is

$$
\delta W=f \cdot \delta X-\tau \cdot \delta q=0
$$

to simplify,

$$
\tau=J^{T} f
$$

where $X$ is displacement in Cartesian-space, $q$ is angular displacement in joint-space and $J$ is Jacobian matrix of manipulator. Then, the force in (5) is represented in virtual forces(spring and damper) adopted as following:

$$
\tau=J^{T}\left(k_{p} \Delta X+k_{d} \Delta \dot{X}\right)+g(q)
$$

where $k_{p, d}$ are virtual spring, damper coefficients and $g$ is gravity function of angle. Virtual spring and damper can be matched in $\mathrm{P}, \mathrm{D}$ terms in control system.

On robot controls, the noteworthy advantages of this Cartesian-space PD control approach are that it is released from annoying singularity problems associated with inverse of the Jacobian matrix and uncertainty problems related to evaluation of physical parameters of robot mechanisms together with actuators and driving mechanisms [5]-[7].

\section{EXPERIMENT}

Our objective is to apply Cartesian-space PD control to the hydraulic driven robots that is already applied to the electrically driven robots. The end-effector displacement for comparison of Joint-space position control with Cartesianspace PD control is a straight-line $\pm 0.3(\mathrm{~m})$ in $X$ axis and 1.4(m) fixed point in $\mathrm{Z}$ axis.

\section{A. Joint-Space Position Control}

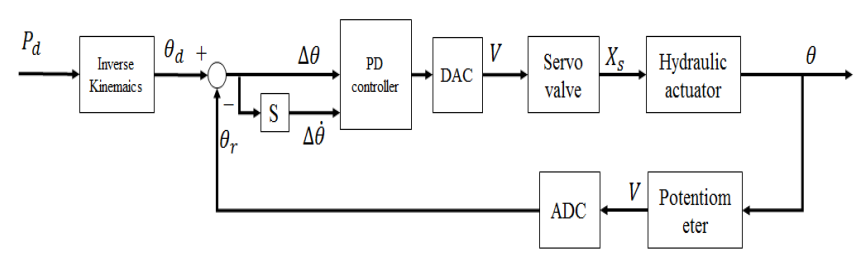

Fig. 4. Position based joint-space PD controller diagram.

The Joint-space position control block is shown in Fig. 4. The Cartesian-space desired position $P_{d}$ generates desired 
angle of each joints. The parameters used in this experiment are given in Table II.

TABLE II: PARAMETERS USED IN THE EXPERIMENT

\begin{tabular}{cccc}
\hline \hline Symbol & Description & Value & Unit \\
\hline$p_{s}$ & Supply pressure & 140 & Bar \\
$f$ & $\begin{array}{c}\text { Control and sampling } \\
\text { frequency }\end{array}$ & 1000 & $\mathrm{~Hz}$ \\
$K_{d x}$ & Damping coefficient in X axis & 2 & - \\
$K_{d z}$ & Damping coefficient in Z axis & 2 & - \\
\hline \hline
\end{tabular}
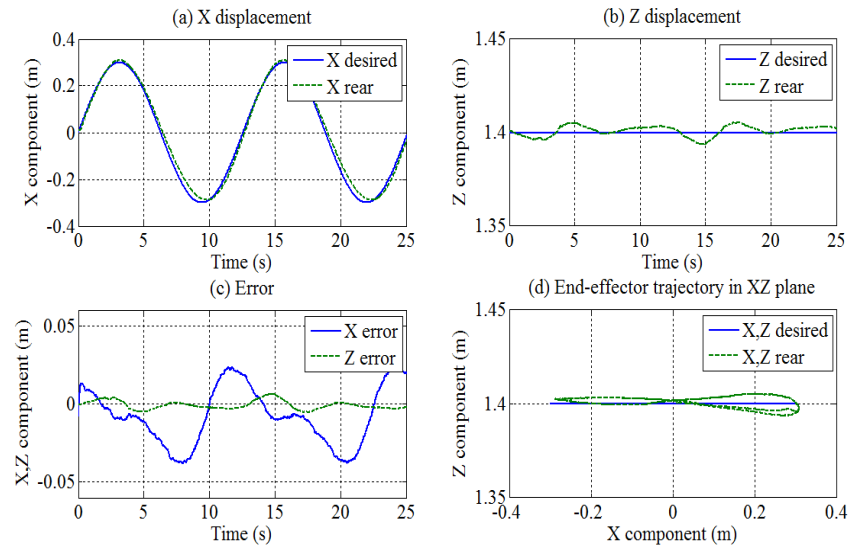

Fig. 5. Experiment results in the case that joint-space position control with sine wave of $\mathrm{X}$ position.
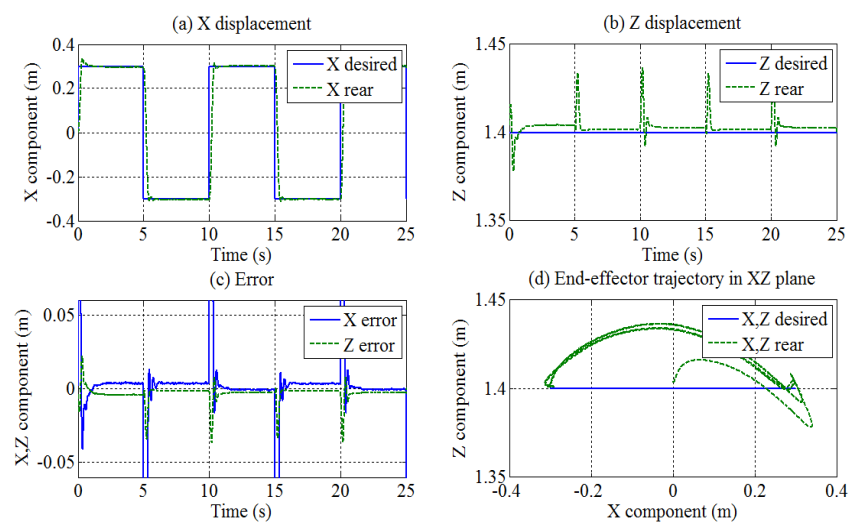

Fig. 6. Experiment results in the case that joint-space position control with square wave of $\mathrm{X}$ position.

Fig. 5 and 6 show the results of experiments with typical joint-space position control. The desired path is given sequentially with step wave experiment. By the time, desired angles of each joint are generated, then each joints has desired angle. It is confirmed that the joints move to target instantly and the position in task space is not taken into account.

\section{B. Cartesian-Space PD Control}

The Cartesian-space PD control block is shown in Fig. 7. The virtual spring and damper at desired position in Cartesian-space pull the end-effector with spring coefficient and damping coefficient. The damping coefficients in $\mathrm{X}, \mathrm{Z}$ axes are fixed as 2 in every experiment.

Fig. 8, 9, 10 and 11 show the results of experiments with Cartesian-space PD control. When the spring coefficient is increasing, the manipulator acts with oscillation like real spring. Fig. 8 and 10 represent the effect of virtual spring coefficient at $X$ axis in sine and step wave. Fig. 9 and 11 represent the effect of virtual spring coefficient at $\mathrm{Z}$ axis in sine and step wave. Each spring coefficient is not too much related to the other.

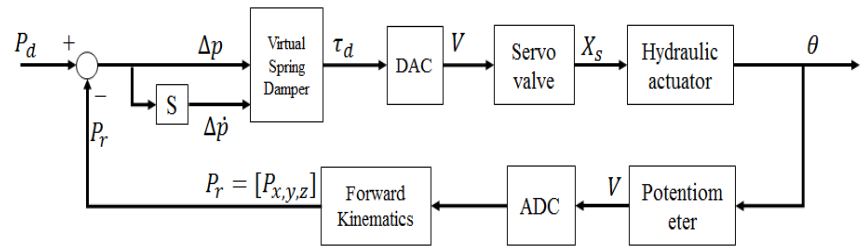

Fig. 7. Cartesian-space PD controller diagram.
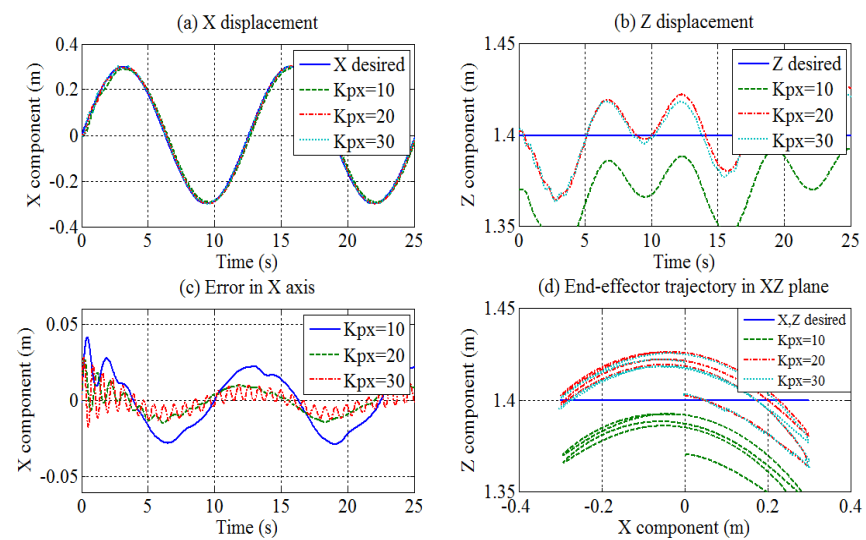

Fig. 8. Experiment results in the case that the effect of spring coefficient in $x$ axis on Cartesian-space PD control with sine wave of $\mathrm{X}$ position.
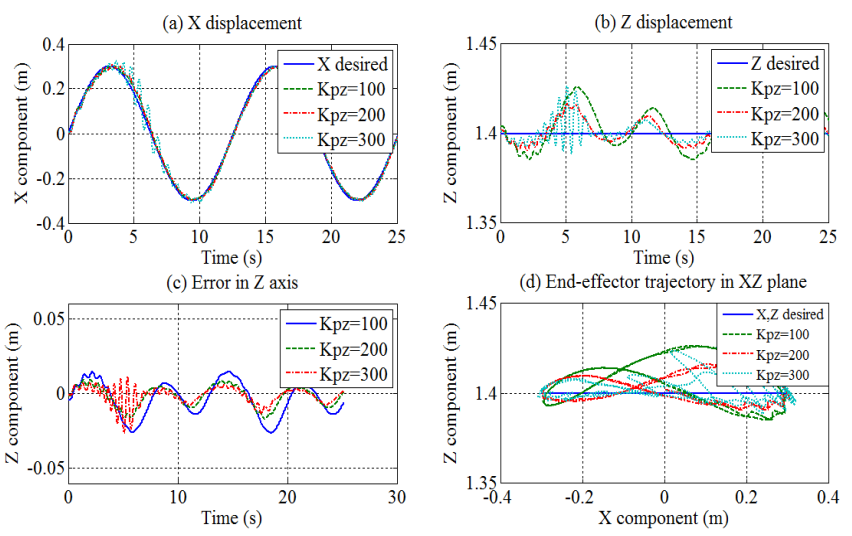

Fig. 9. Experiment results in the case that the effect of spring coefficient in $\mathrm{z}$ axis on Cartesian-space PD control with sine wave of $\mathrm{X}$ position.
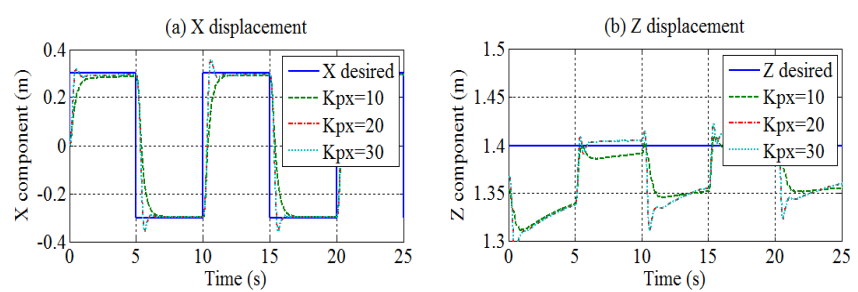

(c) Error in $\mathrm{X}$ axis
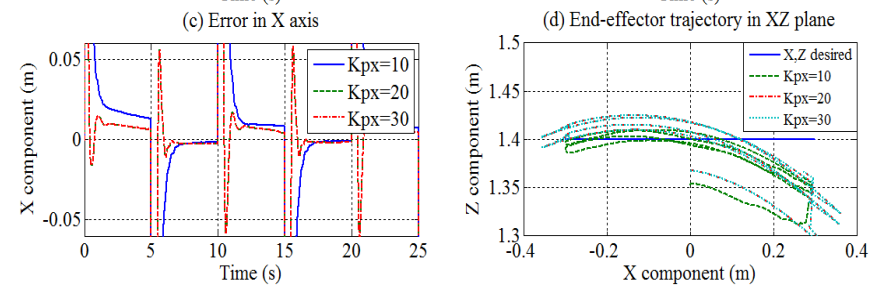

Fig. 10. Experiment results in the case that the effect of spring coefficient in $\mathrm{x}$ axis on Cartesian-space PD control with step wave of $\mathrm{X}$ position. 

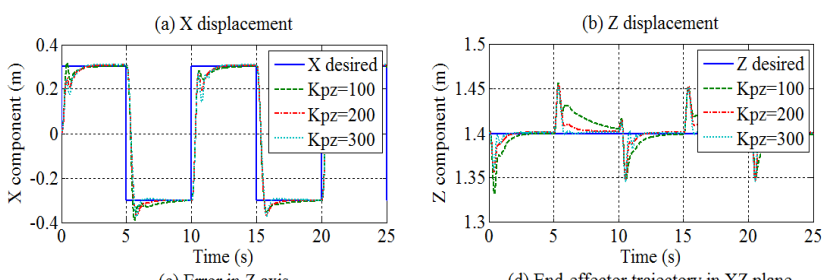

(c) Error in $\mathrm{Z}$ axis
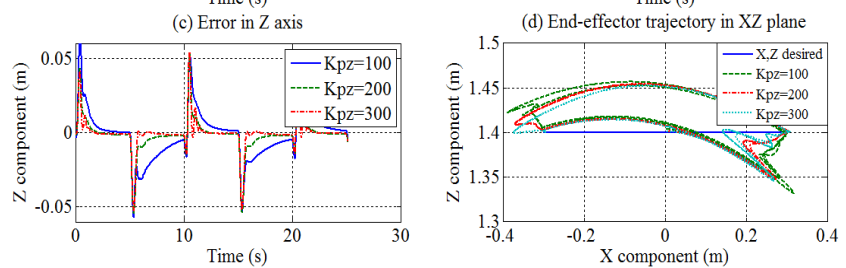

Fig. 11. Experiment results in the case that the effect of spring coefficient in $\mathrm{Z}$ axis on Cartesian-space PD control with step wave of $\mathrm{X}$ position.

\section{Comparison}

The reasonable values of virtual spring and damping coefficients are founded from experiment $\mathrm{A}$ and $\mathrm{B}$ that $k_{p x}$ is 22 and $k_{p z}$ is 270 .
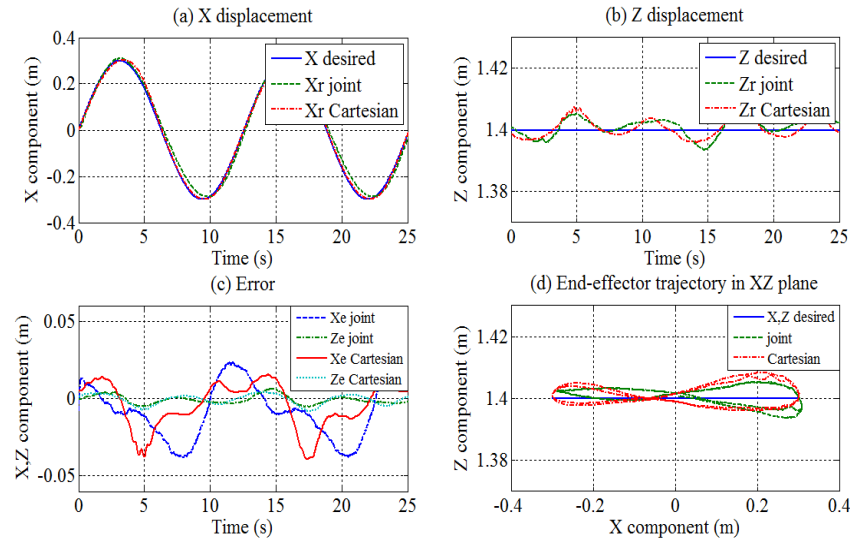

Fig. 11. Comparison joint-space control with Cartesian-space PD control in sine wave of $\mathrm{X}$ position.

Fig. 11 shows comparison of tracking the $0.6(\mathrm{~m})$ of straight-line performance between joint-space control and Cartesian-space control. The errors in this experiment are less than about $\pm 0.03(\mathrm{~m})$.

\section{CONCLUSION}

This paper has described the implementation of Cartesianspace PD control of a hydraulic manipulator. The experiment shows that the torque input can be applied to hydraulic actuator even though hydraulic actuators resemble rather velocity sources than force sources. However, this approach still has challenges that the large gap of spring coefficient between $\mathrm{X}$ axis and $\mathrm{Z}$ axis and gravity compensation problem. It is nearby expected to achieve Cartesian-space control of multi DOF hydraulic manipulator.

\section{REFERENCES}

[1] P. H. Chang and S. J. Lee, "A straight-line motion tracking control of hydraulic excavator system," Mechatronics, vol. 12, pp, 119-138, Feb, 2002.

[2] O. Becker, I. Pietsch, and J. Hesselbach, "Robust task-space control of hydraulic robots," in Proc. IEEE Int. Conf. on Robotics and Automation, 2003, pp. 4360-4365.

[3] J. E. Bobrow and K. Lum, "Adaptive, high bandwidth control of a hydraulic actuator,” ASME J. Dynamic System Meas., Contr., vol. 118, no. 4, pp. 714-720, 1996.

[4] L. Sciavicco and B. Siciliano, Modeling and Control of Robot Manipulators, 2nd ed. Springer, 2004, ch.2, pp. 49-51.

[5] S. Arimoto and M. Sekimoto. "Human-Like movements of robotic arms with redundant DOFs: Virtual spring-damper hypothesis to tackle the bernstein problem," in Proc. IEEE International Conference on Robotics and Automation, pp. 1860-1866, May, 2006.

[6] J. H. Bae, W. Yang, D. Kim, Y. Oh, B. J. You and S. R. Oh. "Robotic arm control inspired by human muscle tension effect under the gravity," in Proc. IEEE Int. Conf. on Robotics and Automation, 2011, pp.1404-1411.

[7] M. Sekimoto and S. Arimoto. "Experimental study on reaching movements of robot arms with redundant DOFs based upon virtual spring-damper hypothesis," in Proc. the 2006 IEEE/RSJ Int. Conf. on Intelligent Robots and Systems, 2006, pp. 562-567.

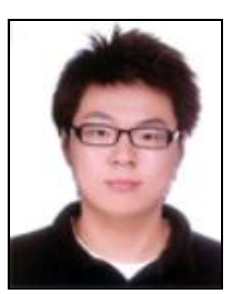

Sang-Uk Chon was born in Korea, 1985. He received the B.Eng. degree in electronic engineering from Hanyang University, Korea in 2012. He is a researcher fellow in Department of Applied Robot Technology, Korea Institute of Industrial Technology.

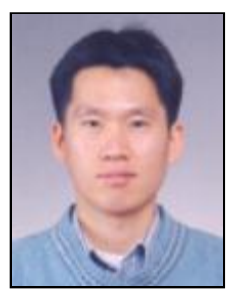

Jung-San Cho was born in Korea, 1976. He received the B.Eng. and M.Eng. degree in electronic control engineering from Kumoh National Institute of Technology, Korea in 2002 and 2004. He is a researcher fellow in Department of Applied Robot Technology, Korea Institute of Industrial Technology.

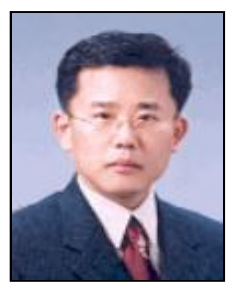

Sang-Deok Park was born in Korea, 1963. He received the B.Eng. in mechanical design engineering from Yeungnam University, Korea in 1988, and M.Eng. and Dr.Eng. in mechanical engineering from Pohang University of Science and Technology, Korea in 1990 and 2000. He is a researcher fellow in the Department of Applied Robot Technology, Korea Institute of Industrial Technology. 